\title{
Von Olympischer Erziehung zu Olympischer Bildung
}

\author{
Stefanie Pietsch
}

\section{Abstract}

Als Einführung und Grundlage für die weiteren Beiträge skizziert dieser Artikel, warum die Olympische Idee nicht nur als Fundament für eine sportliche Werteerziehung gilt, sondern auch ein großes Bildungspotential für eine lebenslange Auseinandersetzung mit Themen und Entwicklungen der Olympischen Bewegung beinhaltet. Dabei werden die erziehungs- und bildungstheoretischen Grundlagen ebenso angesprochen wie die Basiselemente einer Erziehung im und durch Sport. Erziehung, die durch die Einwirkung eines Erziehenden auf eine selbstbestimmte Lebensführung des zu Erziehenden abzielt, bildet die Basis und die Bedingung für die Entfaltung von Bildung. Sie unterstützt die Übernahme grundlegender gesellschaftlicher Werte und Normen und bereitet so den Prozess der Entwicklung einer eigenständigen Persönlichkeit und der Fähigkeit zur individuellen und selbstbestimmten Lebensgestaltung durch Bildung vor. Olympische Erziehung beinhaltet als eine besondere Form der Erziehung im und durch Sport, basierend auf dem Prinzip der kontinuierlichen Arbeit an der Verbesserung der sportlichen Leistungsfähigkeit unabhängig von der Könnens- und Leistungsebene, auch didaktische Prinzipien wie Fairness, soziales Lernen und Gesundheitserziehung. Aufgrund der unmittelbaren Verknüpfung mit dem ambivalenten Konstrukt der Olympischen Spiele reicht eine reine Erziehung nach den olympischen Werten alleine nicht aus. Die Erweiterung des Konstruktes der Olympischen Erziehung hin zu einem dynamischen und ganzheitlichen Ansatz von Olympischer Bildung trägt dazu bei, einen lebensbegleitenden Entwicklungsprozess, durch den kognitive, kulturelle und lebenspraktische Fähigkeiten, aber auch soziale und personale Kompetenzen in Bezug auf olympische Themen erweitert werden, zu initiieren. 


\section{Einleitung}

Olympische Spiele und olympischer Sport üben schon allein aufgrund ihres weltweiten Bekanntheitsgrades sowohl im positiven als auch im negativen Sinn einen nicht zu unterschätzenden Einfluss auf die Einstellungen und Handlungen von Sportler*innen und Zuschauer*innen aus. Dabei geraten die drei ursprünglichen Säulen des Olympismus Sport, Kultur und Erziehung - aufgrund einiger negativer Begleiterscheinungen in Bezug auf die Entwicklung des olympischen Sports häufig in den Hintergrund. Themengebiete wie Kommerzialisierung und Politisierung stehen im Fokus der öffentlichen Aufmerksamkeit. Olympiabewerbungen spalten vor allem in der westlichen Welt die Gesellschaften in Gegner*innen und Befürworter*innen. Kommerzielle Medienspektakel sowie Dopingskandale tragen nicht durchgängig zu einem positiven Gesamtbild der Olympischen Bewegung bei. Daher ist es durchaus verständlich, dass manche Personen dem Begriff einer Olympischen Erziehung, sofern sie ihn denn überhaupt kennen, zunächst zurecht skeptisch gegenüberstehen, da der Begriff „olympisch“ teilweise eben auch eher negativ behaftet ist.

Für Pierre de Coubertin, den Begründer der modernen Olympischen Spiele, war der pädagogische Auftrag einer Olympischen Erziehung, also einer Erziehung mit Hilfe des Sports, ursprünglich eines der zentralen Elemente seiner Olympischen Idee. Dieser von Coubertin formulierte Erziehungsauftrag ist bis heute ein tragendes Element der Olympischen Charta, auch wenn sich die Olympische Bewegung und die daraus resultierenden Spiele seit ihrer Gründung im Jahr 1894 sehr verändert und weiterentwickelt haben. Die zugrundeliegende pädagogische Bedeutung des Olympismus und die Auslegung der Olympische Idee als Erziehungsidee haben aufgrund aktueller Veränderungen und Entwicklungen in den letzten Jahren noch an Brisanz gewonnen und müssen ob der ständig neuen Herausforderungen in einem dynamischen Prozess stetig neu formuliert und hinterfragt werden.

Die entscheidende Frage dabei ist: Eignen sich Olympische Spiele als Bühne und Konstrukt tatsächlich auch heute noch dazu, Kindern und Jugendlichen Werte nahezubringen und zu vermitteln? Ist das vor dem in der Öffentlichkeit wahrgenommenen, vor allem medial vermittelten Bild der Olympischen Spiele mit ihrem Hang zum Gigantismus überhaupt noch zeitgemäß vertretbar? Auf den ersten Blick kann dies einen Erziehenden, unabhängig davon, ob Lehrer*in oder Trainer*in, durchaus in ein Dilemma stürzen, da die in der Regel auf positiven Beispielen basierende pädagogische Wertevermittlung hier auf ein abweichendes und 
ambivalentes Erfahrungswissen trifft. Bei genauerer Betrachtung bietet aber gerade diese Ambivalenz eine hervorragende Möglichkeit zur Auseinandersetzung mit Wertvorstellungen und Normen in einem realistischen und dennoch motivierenden und interessanten Umfeld nicht nur für Kinder und Jugendliche, sondern auch für Erwachsene.

Die in ihrer Verbindung hervorgebrachten olympischen Werte können dabei nach wie vor unter der Voraussetzung der fundierten Beschäftigung mit den einzelnen Aspekten und der konsequenten Umsetzung dieser Werte und moralischen Normen einen wichtigen Beitrag zur Erreichung des Ziels von Frieden, Toleranz und Einvernehmen leisten. Das olympische Gedankengut enthält definitiv Elemente, die gerade wegen der sich teilweise widersprechenden Ausprägungsformen pädagogisch wichtig und moralisch unverzichtbar sind.

Dieser Artikel beschäftigt sich mit der Frage, warum die Olympische Idee neben dem Fundament für eine sportliche Werteerziehung auch ein großes Bildungspotenzial für eine lebenslange Auseinandersetzung mit dem Thema Olympische Spiele bildet. Zunächst werden die erziehungs- und bildungstheoretischen Grundlagen sowie die Basiselemente einer Erziehung im und durch Sport thematisiert. Die Darstellung und Konkretisierung der grundlegenden Bausteine der Olympischen Erziehung und ihre aktuelle fachdidaktische Ausgestaltung führt zu der Erkenntnis, dass eine Erweiterung des Begriffs Olympische Erziehung hin zu Olympischer Bildung sinnvoll ist und dass dieser Schritt das Spektrum der Verbreitung und der Arbeit mit der Olympischen Idee bereichern und erweitern kann. Nur durch eine kritische Beschäftigung mit der aktuellen olympischen Wirklichkeit ist es möglich, einen eigenen fundierten, realistischen und durchaus auch in Teilen kritischen Standpunkt zu entwickeln. Dazu leisten die Beiträge dieses Sammelbands durch eine selbsttätige Auseinandersetzung mit unterschiedlichen olympischen Themengebieten ihren Beitrag.

\section{Erziehung und Bildung}

Um einen Bogen von Olympischer Erziehung zu Olympischer Bildung schlagen zu können bzw. den Begriff der Olympischen Erziehung fundiert zum Begriff Olympische Bildung erweitern zu können, müssen zunächst die zu Grunde liegenden Begriffe Erziehung und Bildung geklärt werden. Was sind die jeweiligen besonderen Merkmale? Was haben Bildung und Erziehung gemeinsam und wie können sie voneinander unterschieden und abgegrenzt werden? 
Bei einer ersten Annäherung wird Erziehung zunächst eher mit Begriffen wie Anleitung, Unterweisung, Ausbildung oder Instruktion in Verbindung gebracht, während man bei Bildung eher an eine Art von Entwicklung, Gelehrtheit, geistigem Überblick oder auch Allgemeinwissen denkt (Seel \& Hanke, 2015). Bereits der Sprachgebrauch verdeutlicht einen ersten großen Unterschied: man wird erzogen, aber man bildet sich selbst. Erziehung und Bildung stehen jedoch definitiv in einem Wechselverhältnis zueinander und leisten einen wichtigen gemeinschaftlichen Beitrag zum individuellen und gesellschaftlichen Entwicklungsvermögen sowie zur Entfaltung einer eigenständigen menschlichen Persönlichkeit.

Erziehung kann definiert werden „als Begriff für alle bewussten Einwirkungen von Menschen, die auf die Entwicklung oder die Veränderung des Wissens und Könnens, dauerhafter Haltungen und Verhaltensformen anderer, insbesondere junger Menschen, gerichtet sind“" (Klafki, 1971, S. 17).

Erziehungsprozesse beinhalten daher - im Gegensatz zu Bildungsprozessen - immer eine Interaktion zwischen zwei oder mehreren Personen, mit dem Ziel, „Lernvorgänge zu unterstützen oder in Gang zu bringen, die zu Disposition und Verhaltensweisen führen, welche von den Erwachsenen [...] als erwünscht angesehen werden" (Brezinka, 1999, S. 26). Es besteht also zu Beginn eines Erziehungsgeschehens immer ein Kompetenzgefälle vom Erziehenden zum Objekt der Erziehung, das mit dem Ziel verringert und überwunden werden soll, dass der Erziehende sich irgendwann selbst überflüssig macht und seine Erziehung, sofern sie erfolgreich verlaufen ist, dem Erzogenen eine selbstbestimmte Lebensführung ermöglicht. Dabei wird Erziehung in der Regel als ein bewusstes, absichtliches und zielgerichtetes Einwirken von Erwachsenen auf die Entwicklung oder Veränderung des Wissens und Könnens junger Menschen angesehen. Durch diese Art der sozialen Interaktion soll durch eine gezielte Änderung des Verhaltens ein intendierter Perspektivwechsel herbeigeführt werden. Erziehung beinhaltet somit all diejenigen Prozesse, die der zu Erziehende im Verlauf des Erziehungsgeschehens unter Anleitung des Erziehenden durchläuft, um nicht nur seine intellektuellen und geistigen, sondern auch seine physischen, emotionalen und sozialen Fähigkeiten zu entwickeln. Neben dieser Erziehung im engeren Sinn, die auch als Persönlichkeitsentwicklung bezeichnet wird, beinhaltet der Erziehungsbegriff im weiteren Sinn zusätzlich auch das Ziel der Sozialisation (Hurrelmann et al., 2008). Unter Sozialisation versteht man dabei das Hineinwachsen in bestimmte vorgegebene Rollen, mit denen eine Person auch immer in einer kontinuierlichen sozialen Interaktion bestimmte Verhaltensformen und -normen 
sowie kulturelle und soziale Inhalte übernimmt. Erziehung im weiteren Sinn beinhaltet daher die Erziehung zu vollwertigen Mitgliedern der sozio-kulturellen Gemeinschaft. Dies erfolgt nicht immer geplant und zielorientiert, sondern geschieht häufig unbewusst ein Leben lang in mitunter äußerst komplexen Interaktionen zwischen einem Individuum und dessen sozialer und kultureller Umwelt. Die prägenden Einflüsse von Familie, Gesellschaft, Staat, Schule und Freundeskreis, sowie aktuelle epochale Strömungen tragen zur jeweiligen Sozialisation und Persönlichkeitsentwicklung des Menschen bei (Hurrelmann et al., 2008).

In Zusammenhang mit Erziehung im engeren bzw. weiteren Sinn, unterscheidet man in der Regel zwischen den beiden Grundformen der intentionalen Erziehung und der funktionalen Erziehung. Intentionale Erziehung ist dabei immer ein zielgerichtetes und absichtsvolles Handeln, mit der in der Regel positiv intendierten Absicht, bei einem zu erziehenden Individuum verschiedene Vorgänge der Person- und Sozialwerdung zu initiieren und zu fördern. Wird dagegen ein negatives Ergebnis angestrebt, so kann intentionale Erziehung in Form einer gezielten, aber nicht offenen Einflussnahme durchaus in die Nähe von Manipulation gerückt werden (Treml, 2000). Die funktionale Erziehung dagegen findet ihren Ausgangspunkt zumeist in lebensweltlichen Situationen sowie gesellschaftlich wirksamen Faktoren wie den Medien oder dem Wertesystem einer Gesellschaft. Funktionale Erziehung tendiert daher in Richtung der Sozialisation, so dass bei dieser Form der Erziehungsvorgang eher nebenbei in der Interaktion mit der sozio-kulturellen Umwelt stattfindet und häufig weder geplant ist noch ein bestimmtes Ziel verfolgt (Raithel et al., 2009). Zwischen funktionaler und intentionaler Erziehung kann die nebenläufige (extensionale) Erziehung angesiedelt werden (Seel \& Hanke, 2015). Zu dieser Form der Erziehung zählt beispielsweise das Verhalten von Erzieher*innen bzw. bestimmte Handlungen, die zwar nicht primär zum Zweck der Erziehung durchgeführt werden, aber durch die Bereitstellung von Umweltgegebenheiten oder die beabsichtigte Vorbildwirkung des Erziehenden als indirekte Einflussnahme zu verstehen sind. Für alle Grundformen der Erziehung sind die jeweiligen erzieherischen Maßnahmen jedoch erst durch die individuelle Mitwirkung des zu Erziehenden tatsächlich realisierbar.

Bildung geht anders als Erziehung von dem sich selbst bildenden Individuum im Dienst der Selbstverwirklichung und des Selbstverständnisses aus und wird häufig als die harmonische Entfaltung aller Kräfte im Menschen beschrieben. Bildung bezieht sich ,auf den in der Person ablaufenden Prozess des „Sichherausbildens“ eines Selbst- und Wertebewusstseins, das auf die Außenwelt gerichtet ist und zeitlich überdauernd das 
Handeln der Person in unterschiedlichen Lebensbereichen beeinflusst“ (Seel \& Hanke, 2015, S. 19). Dabei schreibt Henting (2007) der Bildung die Aspekte der persönlichen, der praktischen und der politischen Bildung zu. Jeder Mensch strebt unabhängig von der Gesellschaft nach persönlicher Bildung, die im Regelfall sehr stark von der Kultur und Schicht bestimmt wird, in der er aufwächst. Praktische Bildung beinhaltet die Aneignung von Wissen und Fertigkeiten, aber auch die Entwicklung von Einstellungen und Verhaltensweisen, die es einem Individuum ermöglichen, sich in der Gesellschaft zurechtzufinden. Konkrete Beispiele für eine praktische Bildung sind nach Lenk (1988, S. 214) unter anderem „Kreativität“, „Flexibilität" und "Selbsterkenntnis" sowie "Sachlichkeit", "Zielstrebigkeit", „interdisziplinäre Offenheit" und "Zivilcourage“. Politische Bildung befähigt ein Individuum dazu, fundierte Entscheidungen angesichts von begrenzten Ressourcen in begrenzter Zeit in Hinblick auf das Gemeinwohl, auf Rechte und Pflichten, aber auch auf Ordnung und Freiheit zu treffen. Eine gebildete Person ist damit im Sinne der kategorialen Bildung nach Klafki (1996) dazu fähig, sich einen Sachverhalt oder Inhaltsbereich sowie neue Kompetenzen im Sinne eines Zusammenschlusses von Wissen und Können selbstständig gedanklich zu erschließen und anzueignen.

Bildung kann daher in einem weiteren Sinn auch als Kompetenzentwicklung ausgelegt werden. Kompetenz, die Aspekte wie Selbst-, Sach-, Methoden- oder Sozialkompetenz beinhaltet, ist dabei zu verstehen als „die bei Individuen verfügbaren oder durch sie erlernten kognitiven Fähigkeiten und Fertigkeiten, um bestimmte Probleme zu lösen, sowie die damit verbundenen motivationalen, volitionalen und sozialen Bereitschaften und Fähigkeiten, um die Problemlösungen in variablen Situationen erfolgreich und verantwortungsvoll nutzen zu können" (Weinert, 2001, S. 27f.). Durch Lernprozesse erlangte Selbstkompetenz befähigt ein Individuum dazu, eigenständig und moralisch handeln zu können, während Sach- bzw. Fachkompetenz sich auf das Wissen und Können in bestimmten Fachgebieten bezieht. Mit Hilfe von Methodenkompetenz können auch komplexe Probleme analysiert und gelöst werden. Sozialkompetenz umfasst die Befähigung zu kommunikativem und kooperativem Handeln. Ein ausgewogenes Verhältnis dieser Kompetenzen zueinander kann daher als „kompetenzorientierte Bildung“ bezeichnet werden (Seel \& Hanke, 2015, S. 22).

Ein gebildeter Mensch verfügt zudem über die Selbstbestimmungsfähigkeit bezüglich individueller Lebensbeziehungen und Sinndeutungen zwischenmenschlicher, beruflicher und ethischer Art. Das heißt, er ist in der Lage, sich zu bestimmten beruflichen, zwischenmenschlichen und religiösen Themen ein eigenes, fundiertes Urteil zu bilden und in diesem 
Sinne zu handeln. Er besitzt Mitbestimmungsfähigkeit im Bereich öffentlicher Angelegenheiten, kann sich in der Gemeinschaft sinnvoll einbringen und besitzt die Fähigkeit zur Solidarität gegenüber denjenigen, denen Selbst- und Mitbestimmung nicht möglich ist (Borst, 2011).

Versucht man nun, Erziehung und Bildung voneinander abzugrenzen, so lässt sich festhalten, dass Erziehung im Prinzip schon kurz nach der Geburt beginnt, während Bildung ein Mindestmaß an Erziehung und persönlicher Reife voraussetzt. Während ein bestimmter Erziehungsabschnitt, wie beispielsweise die Zeit im Kindergarten oder in der Schule, irgendwann abgeschlossen ist, ist Bildung ein lebenslanger, dynamischer und unabschließbarer Prozess, der schließlich zur Selbstbestimmung führen kann. Erziehung liefert somit gewissermaßen die Grundlage für ein anschließendes Gelingen von Bildung. Außerdem kann angeführt werden, dass Erziehung als ein gezieltes Einflussnehmen auf die Personalisation und Sozialisation des zu Erziehenden von außen nach innen wirkt, während sich der Prozess der Bildung ausgehend von den in der Person ablaufenden Prozessen der Entwicklung eines Selbst- und Wertbewusstseins auf das zeitlich überdauernde Handeln der Person in unterschiedlichen Lebensbereichen auswirkt, und somit eher von innen nach außen gerichtet ist. Dabei sind sowohl Erziehung als auch Bildung in der Regel keine punktuellen Ereignisse, sondern unterliegen einem Prozesscharakter. Während der Auslöser für den Erziehungsprozess jedoch in der Regel immer eine Person ist, wird der Bildungsprozess meist durch die sozio-kulturelle Umwelt initiiert. Auch in der Art des Prozessverlaufs gibt es Unterschiede; während Erziehung immer mehr oder weniger stark gelenkt abläuft, ist ein völlig autonomer Ablauf des Bildungsprozesses möglich. Sowohl bei Erziehung als auch bei Bildung handelt es sich jedoch um eine Reihe von kontinuierlichen und dynamischen Verbesserungsprozessen, die nicht umkehrbar sind und eine stetige Erhöhung der Qualität, aber auch die Berichtigung von Fehlern zum Ziel haben. Während aber der Endzustand, also das Ziel jedes Erziehungsprozesses eine mündige Person ist, sollen durch Bildungsprozesse unterschiedliche Handlungskompetenzen entwickelt werden (Seel \& Hanke, 2015).

Nicht in allen Sprachen gibt es diese deutliche Unterscheidung zwischen Bildung und Erziehung anhand zweier sprachlicher Begriffe. Sowohl Erziehung als auch Bildung kann im Englischen beispielsweise mit „education“ übersetzt werden. Was im deutschen Sprachgebrauch konkret unter Erziehung verstanden wird, wird mittels Begriffen wie „upbringing“ oder „training“ spezifiziert, Bildung wird im Englischen als „educated mind“, „formation“ oder „forming“ ausdifferenziert. 
Zusammenfassend lässt sich festhalten, dass Erziehung Handlungen und Maßnahmen beinhaltet, mit deren Hilfe Menschen versuchen, auf die Persönlichkeitsentwicklung anderer Menschen Einfluss zu nehmen, um sie nach bestimmten Wertmaßstäben $\mathrm{zu}$ fördern und $\mathrm{zu}$ formen. Im Gegensatz dazu beinhaltet Bildung die Selbstgestaltung des Menschen im eigenständigen Prozess der Auseinandersetzung mit Inhalten und Werten der Kultur. Während also Erziehung unter konkreter Einwirkung eines Erziehenden auf eine selbstbestimmte Lebensführung abzielt, steht ein ganzheitlicher Bildungsbegriff für den lebensbegleitenden Entwicklungsprozess eines Individuums, in dessen Verlauf er sowohl seine geistigen, kulturellen und lebenspraktischen Fähigkeiten als auch seine sozialen und personalen Kompetenzen im Sinne der selbstbestimmten Gestaltung der eigenen Lebensverhältnisse hin erweitert. Erziehung bildet daher die Grundlage und die Bedingung für die Entfaltung von Bildung. Erziehung begleitet und unterstützt die Übernahme grundlegender gesellschaftlicher Werte und Normen und bereitet so den Prozess der Entwicklung einer eigenständigen Persönlichkeit und der Fähigkeit zur individuellen und selbstbestimmten Lebensgestaltung durch Bildung vor.

\section{Erziehung und Bildung im und durch Sport}

Körperliche Aktivität und die unterschiedlichsten Formen des Sports sind ein fester Bestandteil der kulturell-gesellschaftlichen Lebenswelt. Aufgrund des großen Bedeutungsgehalts in der Umgangssprache ist eine präzise Abgrenzung des Begriffes Sport, der eher „von den historisch gewachsenen und tradierten Einbindungen in soziale, ökonomische, politische und rechtliche Gegebenheiten“ (Röthig, 2003, S. 493) als durch wissenschaftliche Dimensionsanalysen bestimmt wird, nur schwer möglich. Der Lebensbereich Sport spiegelt die Gesellschaft bzw. deren kulturelle Strukturen, Werte und Normen wider und unterliegt einem ständigen Wandel. Formen und Inhalte des Sports sowie Motive und Sinnmuster, die mit Bewegung in Verbindung gebracht werden, stellen sich zunehmend ausdifferenziert dar. Die verschiedenen Formen des Sports repräsentieren daher nicht nur viele unterschiedliche Eigenschaften, Werte und Normen der modernen Gesellschaft, sondern auch deren Fehlentwicklungen und Schattenseiten. Um im DOSB als Sportart aufgenommen zu werden, müssen unter anderem verschiedene Aspekte wie eine „eigene, sportartbestimmende motorische Aktivität“ als Selbstzweck, „die Einhaltung ethischer Werte wie z.B. Fairplay“ und bestimmte organi- 
satorische und strukturelle Voraussetzungen gegeben sein (DOSB, 2018, S. 2 f.).

Sport ist neben seinen vielen anderen Facetten auch in sich ein pädagogisches Konstrukt, da durch die Ausübung der körperlichen Aktivität und die Auseinandersetzung mit den Rahmenbedingungen des Sports die Handlungen und Einstellungen von Sportler*innen (wenn auch nicht immer beabsichtigt oder direkt) beeinflusst werden. Aus pädagogischer Sicht kann Sport als „freiwillige und nicht auf (materielle) Zwecke zielende Lösung von willkürlich geschaffenen Bewegungsaufgaben mit vorrangig körperlichen Mitteln verstanden werden“ (Balz, 2000, S. 8). Die Qualität der Lösung ist dabei verbesserbar und abhängig vom eigenen Können. Die Ausübung von Sport ermöglicht für jeden Menschen eine individuelle und ganz persönliche Begegnung mit der Welt in der Wahrnehmung der eigenen Leiblichkeit. Daher stellen die Entwicklung und Differenzierung des Verhältnisses zum eigenen Körper sowie zu Bewegung und sportlicher Aktivität eine unverzichtbare Aufgabe der allgemeinen Erziehung dar. Bei dieser Form der Leibeserziehung geht es nicht nur um die Erziehung im Sport, also um die spezifische Vorbereitung auf die Anforderungen einer bestimmten Sportart oder allgemein die Verbesserung der körperlichen Leistungsfähigkeit. Vielmehr geht es auch um die Erziehung durch Sport, mit deren Hilfe eine Vielzahl von Erfahrungen und Dispositionen ermöglicht werden soll, die beispielsweise auch Aspekte wie Gesundheit, Kooperation und Fairplay enthalten. Das unmittelbare Erleben solcher Erziehungs- und Bildungsziele im aktiven Sporttreiben ermöglicht vor allem durch eine langfristige Auseinandersetzung mit sportlichen Herausforderungen eine besondere Intensität und Tiefe in Bezug auf die Entwicklung und Entfaltung ebendieser Ziele. Erziehung im und durch Sport und Bewegung beinhaltet also wesentlich mehr als nur eine rein körperliche Ausbildung der motorischen Fähigkeiten. Durch die Ausübung von Sport, mit dessen Hilfe spezifische Erfahrungen und Erlebnisse „am eigenen Leib“ gesammelt werden, erfolgt auch die konkrete Vermittlung von Erziehungs- und Bildungszielen wie Selbstgestaltung und Weltaneignung. So können motorische, kognitive, soziale und emotionale Wirkungsbereiche des Sports wie Gesundheit und Wohlbefinden, aber auch das Erlernen und Anwenden von Regeln und fairem Handeln im sozialen Zusammenleben auf spielerische Art und Weise erprobt werden. Aufgrund der nicht nur theoretischen Unterweisung lassen sich diese über Erlebnis- und Erfahrungssituationen im menschlichen Handeln verankern. Somit beinhalten Bewegungserziehung und Bewegungsbildung nicht nur Möglichkeiten der rein körperlichen Erziehung und Bildung, sondern auch verschiedene wichtige Aspekte der allgemeinen menschlichen 
Bildung. Sie liefern einen wesentlichen Beitrag zur gelungenen Persönlichkeitsentfaltung, ebnen den Weg zu einem gegenseitigen Verstehen und partnerschaftlichen Umgang in einer internationalen Gemeinschaft und sind Ausdruck von Emotionen und Lebensfreude (Prohl, 2008).

\section{Olympische Erziehung}

Wie fügt sich nun der sportpädagogische Ansatz einer Olympischen Erziehung in das Konstrukt der Erziehung im und durch Sport ein? Coubertin, der „Erfinder“ der Olympischen Spiele der Neuzeit spricht im Zuge seiner Erziehungsreform zunächst von Olympismus und Olympischer Pädagogik. Er versteht darunter eine Art Sportlicher Erziehung nach dem Modell des modernen englischen Sports, dem er neben dem Grundsatz der körperlichen Leistungssteigerung entnimmt, dass die moralischen Werte junger Menschen besonders über die individuellen Erfahrungen, die in der Sportpraxis gesammelt werden, entwickelt und auf die eigene Lebenswelt übertragen werden können. Zusätzlich integriert er in sein sportpädagogisches Modell auch Aspekte wie Charakterbildung und Friedenserziehung. Dabei beschränkt er sich in seiner Olympischen Idee nicht nur auf schulische Erziehung, sondern erkennt und propagiert bereits die Bedeutung einer ganzheitlichen Bildung von Körper und Geist, die eine harmonische Erziehung aller Persönlichkeits- und Verhaltensbereiche umfasst, und dies für alle Altersstufen. Das Erziehungsprogramm der Olympischen Erziehung steht für Coubertin für eine allgemeine und Allen zugängliche sportliche Erziehung, die der Motor für das Leben und der Mittelpunkt im Leben der Menschen sein soll. Zweck des Programms ist eine weltweite Realisierung pädagogischer und humanitärer Ziele. Diese Ziele sind die harmonische Bildung des jungen Menschen mit einer geistigen und körperlichen Erziehung als ganzheitlicher Bildung im Sinne der Reformpädagogen sowie die individuelle Selbstvollendung, also ein dauerhafter Lern- und Erziehungsprozess im Sinne der Arbeit an sich selbst (Grupe, 2013). Die Olympischen Spiele selbst sollten dazu beitragen, dieses pädagogische Konzept durch das Vorbild der Athlet*innen bei den Spielen bekannt zu machen und weltweit zu verbreiten. Auch die Olympische Charta des IOC formuliert Olympische Erziehung noch heute als eine der Hauptaufgaben der modernen Olympischen Bewegung. Das IOC benennt konkret drei Werte des Olympismus: „excellence, friendship and respect“, auf denen die gesamte Olympische Bewegung aufgebaut ist (IOC, 2020). Durch die Verbindung des Sports mit Kultur und Erziehung soll in einer pädagogisch-humanistischen Erziehungsidee ein Lebensstil entwickelt 
werden, der zum einen die Freude an der Leistung beinhaltet und zum anderen soziale und humane Werte propagiert (Vedder \& Lämmer, 2008). In Deutschland taucht der Begriff Olympische Erziehung konkret erstmals in den 1970er Jahren auf. Der Sportpädagoge Ommo Grupe (2008) fasste fünf Kernpunkte zusammen, die für Coubertin in seinem Erziehungskonzept (eine genauere Beschreibung hierzu findet sich im Beitrag von Naul) besondere Bedeutung hatten: Das Prinzip der Leib-Seele-Einheit beinhaltet eine harmonische und ganzheitliche Erziehung des Menschen, die über eine rein körperliche Ausbildung hinausgeht. Das Prinzip der individuellen Selbstvollendung ist nicht als Selbstverwirklichung zu interpretieren, sondern beinhaltet einen dauerhaften Lern- und Erziehungsprozess, die beständige Arbeit an sich selbst und das Streben nach menschlicher Vollendung im und durch Sport. Durch das Prinzip des Amateurismus wollte Coubertin sicherstellen, dass nicht materielle Interessen der ausschlaggebende Antrieb für eine sportliche Betätigung im Sinne des olympischen Sports darstellen. Das Prinzip der Ritterlichkeit, oder etwas moderner Fairness, beinhaltet die Bindung des Sportlers an ethische und moralische Regeln und Grundsätze. Durch die Ausübung von Sport soll faires Verhalten erlernt und erlebt werden. Mit dem Prinzip der Friedensidee des Sports will Coubertin den Friedensgedanken auch im Leistungs- und Wettkampfprinzip verankern und im Sinne von Internationalität die Akzeptanz universeller Werte unterstützen. Der Begriff der Olympischen Erziehung muss dabei aufgrund des sich kontinuierlich ändernden Gegenwartsbezugs einer ständigen Überprüfung unterliegen, die Inhalte müssen der aktuellen Lebenswelt von Kindern und Jugendlichen angepasst werden. Naul (2007) ergänzt beispielsweise das Aufgabenfeld der Olympischen Erziehung speziell um die Förderung emanzipatorischer Entwicklungen im und durch Sport. Eine zeitgemäße und moderne Olympische Sporterziehung beinhaltet demnach - basierend auf dem grundlegenden Prinzip des Übens und Trainierens, also der Arbeit an der kontinuierlichen Verbesserung der sportlichen Leistungsfähigkeit unabhängig von der Könnens- und Leistungsebene - auch didaktische Prinzipien wie Fairness, soziales Lernen und Gesundheitserziehung und grenzt sich klar von fachdidaktischen Sportkonzepten wie der reinen Körpererfahrung oder dem Spaßsport ab. Vor allem im Bereich des Schulsports können dabei nach Naul (2007) soziale und moralische Verhaltensweisen gefördert und gefestigt werden. Schülerinnen und Schülern kann ein wichtiges persönliches Knowhow für den Umgang mit den vielfältigen Erscheinungsformen des Sports vermittelt werden. Elemente wie Leistung und die Bereitschaft zur körperlichen Anstrengung werden gezielt mit individuellen und sozialen Lernzielen verknüpft und entfalten sich im gegenseitigen 
Wechselspiel so in komplexer Art und Weise. Dadurch wird Olympische Erziehung zu einer ,integrative[n] Entwicklungsförderung sportlicher, sozialer, moralischer und geistiger Erziehung, die individuelles sportliches Können als Leisten, Wetteifern und Fairplay fördert, um darüber verschiedene positive Sozialerfahrungen und moralische Werthaltungen für den Einzelnen zu entwickeln" (Naul, 2007, S. 106).

Naul und Geßmann gelingt es, die teilweise etwas abstrakt klingenden Formulierungen Coubertins in individuelle Erziehungsaufgaben aus Sicht der sportlichen Erziehung und Sozialisation von Kindern und Jugendlichen zu übersetzen. Geßmann (2004) formuliert als konkrete LehrLernziele für die Olympische Erziehung speziell im Sportunterricht die drei Bereiche Leistung, Fairness und Gegenseitige Achtung. Im Bereich Leistung betont er als eine der Hauptaufgaben die grundsätzliche Arbeit am persönlichen Leistungsfortschritt und der Entwicklung des eigenen sportlichen Könnens. Fairness beinhaltet Aspekte wie die „Bereitschaft, durch Helfen, Unterstützen, Rücksicht-Nehmen [sic] zum Gelingen gemeinsamen Leistens beizutragen" (Geßmann, 2004, S. 145) und in Bezug auf die gegenseitige Achtung stehen Aspekte wie das Akzeptieren und die Anerkennung der Anstrengungen und Leistungen der sportlichen Partner*innen und Gegner*innen im Vordergrund. Naul verbindet in seinem integrierten didaktischen Ansatz unter Einbeziehung verschiedener Ansätze der Olympischen Werte- und Wissensvermittlung die konstitutionellen Grundlagen der Olympischen Charta mit den historisch-pädagogischen Grundlagen und den Erscheinungsformen der olympischen Gegenwart (Naul, 2008). International bekannt ist der lebenswelt-orientierte Ansatz „Be a champion in life!“ der Kanadierin Deanna Binder, deren Ziel es ist, die Olympischen Prinzipien mit den persönlichen soziokulturellen Erfahrungen von Kindern und Jugendlichen sowohl im Sport als auch in anderen Lebensbereichen zu verknüpfen (Binder, 2000). Auch das IOC hat im Rahmen seines Olympic Values Education Programme (OVEP) eine Vielzahl von Lehrmaterialien entwickelt, mit Hilfe derer die Bedeutung von körperlicher und sportlicher Aktivität und das Verständnis für die olympischen Werte und Ideen weltweit gefördert werden sollen (IOC, 2020).

Die didaktischen Arbeiten von Naul, Geßmann, Binder und vielen anderen bekannten Vertreter*innen der Olympischen Erziehung bieten bereits eine große Plattform mit praktischen Anwendungsmöglichkeiten für erzieherische Maßnahmen und Prozesse, die dazu beitragen, wertvolle Erziehungsarbeit im Sinne der Olympischen Idee zu leisten und junge Menschen mittels gezielter Maßnahmen systematisch und gezielt zu fördern. 
Was unterscheidet aber nun Olympische Erziehung von anderen Konzepten einer sportlichen Erziehung? Olympische Erziehung kann grundsätzlich als ein Konzept eingeordnet werden, das auf der Basis von körperlicher Aktivität Leistungs- und Sozialerziehung unter Einbeziehung von Sinn- und Wertfragen miteinander verknüpft (Geßmann, 2004).

\section{Olympische Erziehung}

Olympische Erziehung ist aufgrund der besonderen geschichtlichen Verankerung und der Verbindung mit dem aktuellen und sich beständig weiterentwickelnden Großereignis der Olympischen Spiele eine ganz besondere Form der Erziehung im und durch Sport, die nicht nur einzelne Sinnorientierungen vermitteln will, sondern mit den Aspekten Leistung, Fairness, gegenseitige Achtung, Friedlichkeit und Internationalität einen besonderen umfassenden mehrperspektivischen Erziehungsanspruch beinhaltet.

Sie trägt länder- und kulturübergreifend gerade in der Auseinandersetzung mit aktuellen Trends und Entwicklungen der Olympischen Bewegung zur Sinnbestimmung des Sports in einer modernen Welt bei, ohne dabei einen einzigartigen Erziehungsweg für sich zu beanspruchen.

Olympische Erziehung kann und darf jedoch nicht idealistisch und unkritisch positiv mit dem Großereignis der Olympischen Spiele verknüpft werden. Im Gegenteil, angesichts politischer und kommerzieller Verstrickungen, die in der Öffentlichkeit häufig den olympischen Diskurs dominieren, ist oft auf den ersten Blick wenig Vorbildliches und pädagogisch Sinnvolles erkennbar. Auch wenn derartige negative Aspekte die Olympische Idee und die Ideale der Olympischen Erziehung natürlich nicht völlig außer Kraft setzen, muss deren eigentlicher Sinn, ihre Bedeutung und auch ihre pädagogische Zielsetzung immer wieder neu hinterfragt werden. Olympische Erziehung muss einem Prozess ständiger Weiterentwicklung unterliegen, ihre Werte und Ziele müssen beständig diskutiert und angepasst werden. Trotz der teilweise negativen Konnotation und vereinzelt berechtigten Zweifeln an der Wirksamkeit und Nachhaltigkeit der Vermittlung der olympischen Kernbotschaft gibt es keine andere Großveranstaltung, bei der eine friedliche und kulturelle internationale Vielfalt in einem fairen Wettbewerb so unmittelbar erlebt und vermittelt werden kann. Olympische Erziehung ist also weit mehr als ein weiteres fachdidaktisches Konzept für den Sportunterricht, das dem erziehenden Sportunterricht ähnelt. Es schließt die typische Sporterziehung mit ein und geht in einigen Teilzielen ganz konform mit modernen 
Erziehungszielen wie dem interkulturellen Lernen und der Umwelt- und Friedenserziehung noch darüber hinaus.

\section{Von Olympischer Erziehung zu Olympischer Bildung}

Im Sinne Coubertins sollten Erziehungsziele mittels eines olympischen - an Leistung und Internationalität orientierten - Sports verwirklicht werden, der ein faires Handeln in sportlichen Bewährungssituationen ermöglicht. Dem gegenüber werden die angestrebten Olympischen Ideale häufig durch nationale, politische, kommerzielle und mediale Interessen ad absurdum geführt, so dass Olympiagegner*innen speziell dem olympischen Sport seine pädagogische Bedeutung aberkennen. Aus diesem Blickwinkel betrachtet, reicht eine reine Erziehung nach den olympischen Werten alleine nicht mehr aus. Die olympische Ambivalenz erfordert die Fähigkeit zu einer selbsttätigen und dauerhaften weiterführenden Auseinandersetzung mit olympischen Themen, um die Findung eines fundierten und realistischen persönlichen Meinungsbildes zu ermöglichen, das nicht nur durch den (medial vermittelten) Mainstream bestimmt wird.

Bei genauerer Betrachtung der Prinzipien der Olympischen Erziehung findet sich dieser Ansatzpunkt bereits bei Coubertin wieder. Von den fünf fundamentalen Prinzipien des Olympischen Erziehungsauftrags (Grupe, 2013) kann jedoch nur das erste Prinzip der ganzheitlichen Erziehung als reiner Erziehungsauftrag angesehen werden. Die anderen Prinzipien wie Selbstvollendung, die freiwillige Bindung an Regeln und ethische Grundsätze sowie die Friedensidee erfordern neben den klar formulierten Erziehungszielen für Lernende im Verlauf ihrer weiteren Entwicklung auch den Willen, dieses durch erzieherische Maßnahmen grundlegend entwickelte Potenzial selbsttätig zu entfalten und weiterzuentwickeln sowie selbstständig und mündig damit umzugehen. Aufgrund der Komplexität und der untrennbaren Vernetzung der Olympischen Bewegung mit aktuellen Themen und Entwicklungen, die eine lebenslange Auseinandersetzung mit wechselnden Perspektiven ermöglicht, beinhaltet die Olympische Idee über das Ideal einer sportlichen Erziehung mit differenzierten Erziehungsaspekten für Kinder, Jugendliche und Olympiateilnehmer*innen hinaus die Grundlage für eine lebenslange sportliche Bildung. Interessante Ansatzund Verknüpfungsmöglichkeiten hierfür bietet unter anderem die Agenda 2020+5 (IOC, 2021). Olympische Erziehung endet also nicht automatisch mit dem Ende der Kindheit und Jugend, sondern geht fließend in Olympische Bildung über. Gerade weil die Olympische Idee aufgrund der olympischen Realität kein abstraktes und idealisiertes pädagogisches Vorzeigemo- 
dell ist, birgt das Konstrukt der Olympischen Bildung als Erweiterung der Olympischen Erziehung viele interessante Möglichkeiten und Chancen für eine komplexe und differenzierte Beschäftigung sowie eine kritische Auseinandersetzung mit der olympischen Kernidee auch im Erwachsenenalter. Erste Ansätze für diese weiter gefasste Sichtweise erwähnen bereits Naul et al. (2017, S. 177, 184), die in diesem Zusammenhang die Begriffe "formal teaching" und „formal olympic education" im Sinne von Olympischer Erziehung in der Schule und ,informal olympic learning“ im Sinne von Olympischer Bildung in Sportvereinen verwenden.

Wie genau kann nun diese Erweiterung hin zu Olympischer Bildung vonstattengehen?

Olympische Erziehung kann durch ihre vielfältigen methodisch-didaktischen Ansätze dazu beitragen, positiv auf die Persönlichkeitsentwicklung junger Sportler*innen Einfluss zu nehmen, und sie nach bestimmten Wertmaßstäben entsprechend der Olympischen Idee zu fördern sowie auf eine selbstbestimmte Lebensführung hinzuarbeiten. Olympische Erziehung bildet damit gewissermaßen die Grundlage und die Bedingung für die Entfaltung von Olympischer Bildung.

Im Gegensatz zur Olympischen Erziehung im engeren Sinn, bei der beispielsweise olympische Werte mit Hilfe bestimmter Unterrichtsmaterialien oder Arbeitsaufgaben gefördert werden oder auch der Olympischen Erziehung im weiteren Sinn, bei der die Olympische Idee zum Beispiel bei der Durchführung eines olympischen Tages selbst erlebt werden kann, steht ein dynamischer und ganzheitlicher Ansatz der Olympischen Bildung für einen lebensbegleitenden Entwicklungsprozess des Menschen, der dazu beiträgt, seine kognitiven, kulturellen und lebenspraktischen Fähigkeiten, aber auch seine sozialen und personalen Kompetenzen in Bezug auf olympische Themen zu erweitern und weiterzuentwickeln. Mit Hilfe olympischer Bildungsprozesse kann es gelingen, die Selbstgestaltung von Menschen jeden Alters im Prozess der Auseinandersetzung mit olympischen Inhalten und Werten zu unterstützen. Da die Olympische Idee mit den Olympischen Spielen kein punktuelles und einmalig durchgeführtes Event, sondern ein geschichtlich fundiertes langlebiges, sich ständig weiter entwickelndes Phänomen darstellt, lädt sie wie kaum etwas Anderes zu einer beständigen und dauerhaften kritischen Auseinandersetzung mit olympischen Themen ein.

Auch für Olympische Erziehung und Olympische Bildung gilt: man wird „olympisch erzogen“, und zwar mithilfe der unterschiedlichen didaktisch-methodischen Ansätze, die vielfältige Möglichkeiten der Auseinandersetzung mit geschichtlichen, körperlichen und moralischen Aspekten der Olympischen Idee altersgerecht aufbereiten und vermitteln und ein 
persönliches Erleben der verschiedenen Inhalte wie Fairness, Leistung oder gegenseitige Achtung unmittelbar erlebbar machen. Ab einem gewissen Alter, spätestens mit dem Verlassen der Schule endet jedoch der Abschnitt einer Olympischen Erziehung. Eine weiterführende eigenständige Beschäftigung mit olympischen Themen geht in den Bereich des „sich olympisch Bildens“ über. Dabei handelt es sich jedoch nicht um einen abrupten Wechsel von olympischer Erziehung zu olympischer Bildung, der Übergang erfolgt fließend. Die Grundsätze und Werte einer Olympischen Erziehung verlieren nicht an Bedeutung, im Gegenteil, diese grundlegend vermittelten Prinzipien werden im Verlauf einer selbsttätigen Olympischen Bildung weiterentwickelt und individuell ausdifferenziert. Olympische Erziehung und Olympische Bildung stehen daher immer in einem Wechselverhältnis zueinander. Olympische Bildung kann dabei nicht nur zur Herausbildung eines generellen Selbst- und Wertebewusstseins beitragen, sondern auch zu den einzelnen Teilbereichen einer persönlichen, praktischen und politischen Bildung. Die fundierte Auseinandersetzung mit olympischen Themen unterstützt die Aneignung von Wissen ebenso wie die Entwicklung von differenzierten Einstellungen und Verhaltensweisen, die auf den Prinzipien der Olympischen Bewegung beruhen. Damit trägt Olympische Bildung auch zur Kompetenzentwicklung des Individuums bei. Die kritische Auseinandersetzung mit olympischen Themen, aber auch das Erleben olympischer Werte bereichern nicht nur das „olympische Wissen“, sondern auch die Fähigkeit zu eigenständigem, moralischem, kommunikativem und kooperativem Handeln.

Konkret eignen sich aktuelle olympische Themenbereiche wie Nachhaltigkeit, Kommerzialisierung, Gigantismus und Doping, aber auch die Auseinandersetzung mit der Olympischen Vergangenheit und deren Entstehungsgeschichte oder die Entwicklung der Paralympischen Bewegung aufgrund der Ambivalenz der olympischen Bewegung und ihrer Strukturen hervorragend als vielschichtiges Bildungsfeld. Gerade in Zeiten der sozialen Medien, in denen eine Fülle von ungeprüften Informationen ungefiltert und häufig mit unklarer Quellenlagen sehr schnell sehr viele Menschen erreicht, ist Olympische Bildung lebenslang von enormer Bedeutung, um aktuelle Entwicklungen kompetent beurteilen und einschätzen zu können. Vor allem in diesem Bereich müssen weitreichende Bildungsprogramme der nationalen und internationalen Komitees ansetzen. Dies gilt nicht nur für olympische Athlet*innen, sondern auch für alle im Umfeld der Olympischen Spiele und der Olympischen Bewegung tätigen Personen und die interessierte Allgemeinheit. Schlüsselkompetenzen einer zeitgemäßen Allgemeinbildung wie Selbstbestimmungs-, Mitbestimmungs- und Solidaritätsfähigkeit können 
anhand der Beschäftigung mit Olympia erworben und vertieft werden (und ermöglichen so unter anderem auch eine kritische und fundierte Auseinandersetzung mit der Olympischen Idee im Allgemeinen und der Olympischen Erziehung im Speziellen). Eine moderne Sichtweise von Olympischer Erziehung muss demnach perspektivisch den Ansatz der Olympischen Bildung integrieren, durch die sich beständig neue und aktualisierte Perspektiven und Betrachtungsweisen im Hinblick auf olympische Themen ergeben. Olympische Bildung bietet die Basis für eine glaubhafte und kritische Auseinandersetzung mit den sich beständig weiterentwickelnden Darstellungsformen der olympischen Werte. Sie trägt dazu bei, ein realistisches Verständnis für die Gesamtheit und Komplexität der Olympischen Idee zu entwickeln und diese Erkenntnisse auf die eigene Lebenswelt zu übertragen, um diese kritisch zu hinterfragen. Somit stellen Olympische Erziehung und in ihrer Erweiterung auch eine lebenslange Olympische Bildung so aktuell wie nie ein zentrales Element der Erziehung im und durch Sport sowie der Alltagsbildung durch Bewegung, Spiel und Sport dar.

\section{Bildungsbeiträge zu einer neuen Perspektive auf Olympia}

Basierend auf diesen pädagogischen Grundlagen in Bezug auf Olympische Erziehung und Olympische Bildung ist es das Hauptanliegen dieses Buches, aktuelle Themenbereiche in Zusammenhang mit Olympia inhaltlich zu skizzieren und ihr Bildungspotenzial aufzuzeigen. Ziel ist es, den interessierten Leser*innen fundierte und realistische Einblicke zu ermöglichen und ihnen so die Gelegenheit zu bieten, sich in Bezug auf olympische Themen weiterzubilden. In den drei Kapiteln OLYMPIC BASICS: Geschichtliche, bildungstheoretische und sozialwissenschaftliche Grundlagen; OLYMPIA AKTUELL: Aktuelle olympische Fragestellungen und Entwicklungen und OLYMPIA INSIDE: Blickwinkel auf Olympia stellen renommierte Expert*innen für ihre jeweiligen Fachbereiche neue Blickwinkel und Perspektiven der Betrachtungsweise der Olympischen Idee und der Olympischen Spiele vor. Im ersten Teil dieses Buches OLYMPIC BASICS wird olympisches Grundwissen mit einem Schwerpunkt auf unterschiedliche geschichtliche, bildungstheoretische und sozialwissenschaftliche Aspekte vermittelt. Mythos und Realität der Olympischen Spiele der Antike werden ebenso hinterfragt wie die Olympische Idee Coubertins, die zur Einführung der Olympischen Spiele der Neuzeit führte. Dieses Kapitel liefert auch eine Antwort auf die Frage, warum die Olympischen Spiele der Neuzeit wesentlich umfassender sind, als nur ein beliebiges 
internationales Sportereignis. Nach der historischen Einordnung werden Entwicklungen der Olympischen Bewegung, die diese vielfältiger werden lassen, am Beispiel der Deaflympics, Paralympics und Special Olympics sowie die Entwicklung des olympischen Wettkampfprogramms hinterfragt und eingeordnet. Neue Formate mit modernen Sportarten sowie die Bedeutung von Wertvorstellungen und Moral stehen in diesem Teil des Buches ebenso im Fokus wie Genderfragen im olympischen Sport.

Im Kapitel OLYMPIA AKTUELL finden sich Beiträge von Expert*innen, die aktuelle sportpolitische Herausforderungen sowie die Inszenierung der Spiele als Megaevent zum Gegenstand haben. Die Autor*innen gehen auf weitreichende ökonomische Aspekte ebenso ein wie auf die Darstellung von Olympia in den Medien und im digitalen Zeitalter. Auch das Konzept und die Entwicklung der anfangs nicht unumstrittenen Youth Olympic Games wird aufgezeigt. Ein wichtiger Bereich dieses Kapitels widmet sich speziell den Olympischen und Paralympischen Spielen in Deutschland. Hätten Olympische und Paralympische Spiele tatsächlich aus gesellschaftspolitischer Perspektive und aus der Sicht des organisierten Sports einen Mehrwert für die Gesellschaft? Ein Rückblick auf vergangene Bewerbungen gibt Einblick in die Chancen und Risiken zukünftiger Bewerbungen. Während in den ersten beiden Kapiteln Wissenschaftler*innen und Fachleute über neue Perspektiven der Olympischen Bildung nachdenken, kommen im Kapitel OLYMPIA INSIDE diejenigen zu Wort, die aus erster Hand aufgrund von konkreten Erfahrungen aus unterschiedlichen Blickwinkeln vom Bildungspotenzial der Olympischen Bewegung und der Olympischen Idee berichten können. Olympia- und Paralympics-Teilnehmer*innen schildern aus ihrer jeweiligen Perspektive als Teilnehmer*innen, Trainer*innen, Funktionär*innen oder auch Seelsorger*innen ihre ganz persönlichen Eindrücke und Erlebnisse.

\section{Literatur:}

Balz, E. (2000). Sport oder Bewegung - eine Frage der Etikettierung? dvs-Informationen, 15(4), 8 - 12 .

Binder, D. (2000). Be a champion in life! A book of activities for young people based on the joy of participation and on the important messages of the Olympic idea. An international teachers' resource book for schools. Athens: FOSE.

Borst, E. (2011). Theorie der Bildung. Eine Einführung. Pädagogik und Politik (Band 2). Baltmannsweiler: Schneider Verlag Hohengehren. 
Brezinka, A. (1999). Von der Pädagogik zur Erziehungswissenschaft (2. Aufl.). Weinheim: Beltz.

DOSB (2018). Aufnahmeordnung des DOSB. Abgerufen am 28.03.2021 unter https://cdn.dosb.de/user_upload/www.dosb.de/uber_uns/Satzungen_und_ Ordnungen/aktuell_Aufnahmeordnung_2018_.pdf

Geßmann, R. (2004). Olympisches Menschenbild und schulische Sportdidaktik. In NOK (Hrsg.), Olympische Erziehung - eine Herausforderung an Schulpädagogik und Schulsport (S. 1131-1153). Sankt Augustin: Akademia.

Grupe, O. (2008). Die Olympische Idee ist eine „Erziehungsidee“ - das sollte sie auch bleiben. Olympisches Feuer, 4/5, 55-60.

Grupe, O. (2013). Die Olympische Idee ist eine „Erziehungsidee“. In A. Hofmann, \& M. Krüger (Hrsg.), Olympia als Bildungsidee. Beiträge zur Olympischen Geschichte und Pädagogik (S. 9 - 22). Wiesbaden: Springer.

Hurrelmann, K., Grundmann, M. \& Walper, S. (Hrsg.). (2008). Handbuch Sozialisationsforschung (7. Aufl.). Weinheim: Beltz-Juventa.

IOC (2020). Olympic values education programme. Abgerufen am 12.12.2020 unter https:/www.olympic.org/olympic-values-and-education-program

IOC (2021). IOC Executive Board proposes Olympic Agenda 2020+5 as the strategic roadmap to 2025. Abgerufen am 08.04.2021 unter https://www.olympic.org/ news/ioc-executive-board-proposes-olympic-agenda-2020-plus-5-as-the-strategicroadmap-to-2025

Klafki, W. (1971). Erziehungswissenschaft als kritisch-konstruktive Theorie: Hermeneutik - Empirie - Ideologiekritik. Zeitschrift für Pädagogik, 17(3), 351366.

Klafki, W. (1996). Neue Studien zur Bildungstheorie und Didaktik: Zeitgemäße Allgemeinbildung und kritisch-konstruktive Didaktik. Weinheim: Beltz.

Lenk, H. (1988). Bildungswerte im informations- und systemtechnologischen Zeitalter. In W. Böhm \& M. Lindauer (Hrsg.), „Nicht Vielwissen sättigt die Seele“. Wissen, Erkennen, Bildung, Ausbildung heute. Stuttgart: Klett.

Naul, R. (2007). Olympische Erziehung. Edition Schulsport (Band 7). Aachen: Meyer \& Meyer.

Naul, R., Krüger, M., Geßmann, R. \& Wick, U. (2017). Germany. Formal Olympic education at schools and informal Olympic learning in sport clubs. In R. Naul, D. Binder, A. Rychtecky \& I. Cuplan (Hrsg.), Olympic Education. An international review. (S. 177-191). London and New York: Routledge.

Prohl, R. (2008). Erziehung mit dem Ziel der Bildung: Der Doppelauftrag des Sportunterrichts. In H. Lange \& S. Sinning (Hrsg.), Handbuch Sportdidaktik (S. 40-53). Balingen: Spitta.

Raithel, J., Dollinger, B. \& Hörmann, G. (2009). Einführung Pädagogik. Begriffe, Strömungen, Klassiker, Fachrichtungen (3. Aufl.). Wiesbaden: VS Verlag für Sozialwissenschaften.

Röthig, P., Prohl, R. et al. (Hrsg.) (2003). Sportwissenschaftliches Lexikon. Schorndorf: Hofmann. 
Seel, N. M., \& Hanke, U. (2015). Erziehungswissenschaft. Lehrbuch für Bachelor-, Master- und Lebramtsstudierende. Berlin Heidelberg: Springer.

Treml, A. K. (2000). Allgemeine Pädagogik. Grundlagen, Handlungsfelder und Perspektiven der Erziehung. Stuttgart: Kohlhammer.

Vedder, C. \& Lämmer, M. (2008). Olympische Charta 2014 (Deutsche Übersetzung). Frankfurt/Main: Deutsche Olympische Akademie.

von Henting, H. (2007). Klassischer Bildungsbegriff und pragmatisches Bildungsverständnis. In R. Wernstedt \& M. John-Ohnesorg (Hrsg.), Der Bildungsbegriff im Wandel, Verführung zum Lernen statt Zwang zum Büffeln (S.13-17). Berlin: Friedrich-Ebert-Stiftung.

Weinert, F. E. (Hrsg.) (2014). Leistungsmessung in Schulen. Weinheim und Basel: Beltz. 